\title{
Optimization of Arginine Fermentation Culture Medium on Recombinant Strain of Corynebacterium glutamicum
}

\author{
TIAN Hong-ming ${ }^{1,2, a}$ \\ ${ }^{1}$ College of Biological Science and Engineering, South China University of Technology, Guangzhou \\ 510006, China \\ ${ }^{2}$ Guangdong Key Laboratory of Fermentation and Enzyme Engineering, Guangzhou 510006, China \\ atian.hm@mail.scut.edu.cn
}

Keywords: carbon; Nitrogen; Arginine; Fermentation

\begin{abstract}
In the recombinant strain Corynebacterium glutamicum ATCC14067-T18-argB, the key kinase (N-acetylglutamate kinase) was expressed with the PEC-T18mob2 plasmid. The ability of producing L-arginine was increased. Aimed at improving the production of L-arginine further, several factors include soya peptone, glucose, urea, calcium carbonate and liquid volume were studied. Based on the one-variable-at-a-time experiment of the fermentation medium, three main significant factors of the soya peptone, urea and glucose were screened by the Box-Bohnken design. After the optimization of fermentation medium by the Box-Bohnken results showed optimal medium containing of soya peptone $9.0 \mathrm{~g} / \mathrm{L}$, glucose $8.0 \mathrm{~g} / \mathrm{L}$ and urea $8.0 \mathrm{~g} / \mathrm{L}$. The mutant ATCC14067-T18-argB could accumulate 10.77g/L L-arginine after $72 \mathrm{~h}$ fermentation. Compared with the initial fermentation medium, the production on average increased nearly 3 times. The result of comparative experiment showed that the increase of urea which is the nitrogen and the decrease of glucose which is the carbon source made contribution to the increase of L-arginine.
\end{abstract}

\section{Introduction}

L-arginine is the final product of L-arginine biosynthetic pathway, semi-essential basic amino acid for humans and animals and essential amino acid for young animals [2]. L-arginine is involved in many areas of vital activity, including ammonia detoxification, hormone secretion and immune system, etc. It has important medical value in the treatment of cardiovascular, nervous and endocrinic diseases, etc.[1].

The synthetic pathway of L-arginine production strains can be divided into two categories. In the synthetic pathway of Escherichia coli, Bacillus subtilis and other microbes, glutamate-synthetic Nacetylglutamate and $\mathrm{N}$ - acetylornithine-synthetic ornithine are catalyzed by $\mathrm{N}$-acetylglutamate synthase and $\mathrm{N}$-acetylornithine synthase respectively. While in the metabolic pathway of Corynebacterium glutamicum, yeast and other microbes, these two reactions are catalyzed by conjugation reaction between $\mathrm{N}$-acetylglutamate-ornithine acetyl transferase. There exists an acetyl cycle reaction from glutamate to ornithine [3] The breeding methods of L-arginine production bacteria are usually mutation breeding and genetic engineering. With C.glutamicum DSM1412 as the original strain, after step-by-step mutagenization and breeding with ultraviolet and N-methyl-N' -nitro-N-nitrosoguanidine, as well as resistance breeding using NG-nitro-L-arginine methyl ester, the Chinese Academy of Sciences obtained a L-arginine production strain. The yield of L-arginine from this strain was up to 31g/L[4]. Ginesy et al. excessively expressed argA214 or argA215 genes in three strains of e. coli whose argR (encoding an arginine responsive repressor protein), speC, speF (encoding ornithine decarboxylases) and adiA (encoding an arginine decarboxylase) were knocked out and found that the constructs produced between 1.94 and $3.03 \mathrm{~g} / \mathrm{L}$ arginine. In the knockout argA gene recombinant bacteria argP216 and argA214 or excessive expression genes argA214 and argO, the yield of L-arginine reached $7.95 \mathrm{~g} / \mathrm{L}$ and $11.64 \mathrm{~g} / \mathrm{L}$ respectively and increased 2-3 times than argA knockout bacteria[5]. Based on C.glutamicum ATCC 21831 as the original strain, Park et al. knocked out the repressor protein gene argR in the synthetic pathway of L-arginine, excessively expressed 
genes in the biosynthetic pathway of L-arginine and L-arginine transporter and finally resulted in the production of $92.5 \mathrm{~g} / \mathrm{L}$ L-arginine at a laboratory level[6].

In this study, the author uses the recombinant strain ATCC14067-T18-argB stored in the lab to produce L-arginine. The strain enhanced the yield of L-arginine by excessively expressing the key kinase NAGK in the synthetic pathway of L-arginine. To further improve the yield, response surface method is adopted to optimize the fermentation medium.

1. Materials and Methods

1.1 Bacterial strains and Culture medium

L-arginine production bacteria ATCC14067-T18-argB (C.glutamicum ATCC14067 with plamid PEC-T18mob2-ArgB) was used as the construct in this lab.

LBG medium: $10 \mathrm{~g} / \mathrm{L}$ peptone, $5 \mathrm{~g} / \mathrm{L}$ yeast powder, $5 \mathrm{~g} / \mathrm{L}$ glucose and $10 \mathrm{~g} / \mathrm{L} \mathrm{NaCl}$; seed medium: 20 g/L glucose, 20 g/L peptone, 1.5 g/L K2HPO4, 0.5 g/L KH2PO4 and 0.5 g/L MgSO4; initial fermentation medium: $20 \mathrm{~g} / \mathrm{L}$ soy peptone, $150 \mathrm{~g} / \mathrm{L}$ glucose, $3 \mathrm{~g} / \mathrm{L}$ urea, $30 \mathrm{~g} / \mathrm{L} \mathrm{CaCO} 3,0.7 \mathrm{~g} / \mathrm{L}$ K2HPO4 and 0.5 g/L MgSO4; optimum fermentation medium: 9 g/L soy peptone, 80 g/L glucose, 9 g/L urea, 30 g/L CaCO3,0.7 g/L K2HPO4 and 0.5 g/L MgSO4.

\subsection{Experimental Method}

\subsubsection{Activation of bacteria}

Bacteria stored in a cryogenic vial at $-80^{\circ} \mathrm{C}$ was cultured at $30^{\circ} \mathrm{C}$ for $24 \mathrm{~h}$ on a tetracyclineresistant $\mathrm{LB}$ solid medium and streak transferred 3 times to get activated strains. Single colony was screened from the activated strains to be inoculated to the activated medium and cultured at $30^{\circ} \mathrm{C}$, $250 \mathrm{rpm}$ for $24 \mathrm{~h}$.

\subsubsection{Culture method}

The activated strains were inoculated to a seed medium with tetracycline at a final concentration of $5 \mu \mathrm{g} / \mathrm{mL}$ at $30^{\circ} \mathrm{C}, 250 \mathrm{rpm}$ for 0D600 30 or so and the start OD was controlled as 0.1 . The bacteria were inoculated to a fermentation medium with tetracycline at a final concentration of $5 \mu \mathrm{g} / \mathrm{mL}$ at $30^{\circ} \mathrm{C}, 250 \mathrm{rpm}$ for $72 \mathrm{~h}$. Fermentation broth was collected. The yield of L-arginine was measured using high performance liquid chromatography (HPLC).

1.2.3 Determination of L-arginine content in the fermentation broth [7]

The L-arginine content was determined by HPLC precolumn derivatization. Derivatization method: $2 \mathrm{~mL}$ fermentation broth was centrifuged at $10000 \mathrm{rpm} / \mathrm{min}$ for $1 \mathrm{~min}$. $150 \mu \mathrm{L}$ supernatant was collected and added to a $2 \mathrm{~mL}$ centrifuge tube. $150 \mu \mathrm{L}$ ready-made derivatized buffer solution was added (4.2g sodium bicarbonate was dissolved with water to $100 \mathrm{~mL}, \mathrm{pH}$ adjusted to 9.0). After shaking well, $150 \mu \mathrm{L}$ derivatized solution was added (1mL 2,4-Dinitrofluorobenzene was put in a $100 \mathrm{~mL}$ volumetric flask and diluted with acetonitrile to scale) and shaken well. The centrifuge tube was put in a $60^{\circ} \mathrm{C}$ water-bath heater and allowed to stand in the dark for $60 \mathrm{~min}$. After that, cooled to room temperature. $1050 \mu \mathrm{L}$ balanced solution was added (3.4g disodium hydrogen phosphate was weighed accurately, put in a $500 \mathrm{~mL}$ volumetric flask and dissolved with water. $\mathrm{pH}$ was adjusted to 7.0 using $0.1 \mathrm{~mol} / \mathrm{L}$ sodium hydroxide and diluted to scale). After standing for $10 \mathrm{~min}$, centrifuged at $10000 \mathrm{rpm} / \mathrm{min}$ for $1 \mathrm{~min}$, filtered with $0.22 \mu \mathrm{m}$ Hydrophilic Durapore Membrane and set aside. Agilent 1100 HPLC, Agilent Hypersil BDS-C18 chromatographic column, UV detector (254nm) were applied for detection. Mobile Phase A: $0.05 \mathrm{~mol} / \mathrm{L}$ sodium acetate buffer solution, only $1 \%$ N,N-Dimethylformamide pH6.8. Mobile Phase B: $50 \%$ acetonitrile buffer solution. The velocity of mobile phase was $1.0 \mathrm{~mL} / \mathrm{min}$. The column temperature was $43^{\circ} \mathrm{C}$

1.3 Experimental design

1.3.1 Single-factor optimization

Glucose, calcium carbonate, urea and soy peptone were screened for single-factor optimization. The evaluation index was the concentration of L-arginine in fermentation broth. In each group, all treatments were in triplicate and averaged. The experiment design is shown in Table 1.

Table 1 Signal factor experiment design

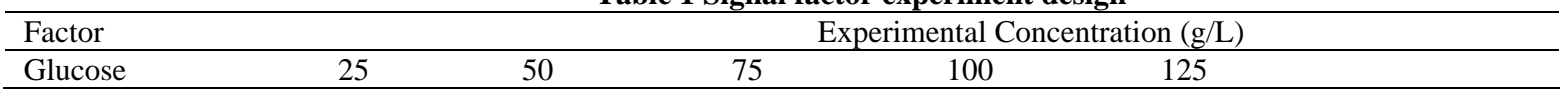




\begin{tabular}{lllllll}
\hline Calcium carbonate & 10 & 20 & 30 & 40 & 50 & \\
Urea & 1 & 3 & 5 & 7 & 9 & 12 \\
Soy peptone & 0 & 2 & 6 & 10 & 20 & 30 \\
\hline
\end{tabular}

\subsubsection{Response surface analysis}

According to Placket-Boxhenm' s central composite design, 3 levels were selected from 3 factors identified in two-level designs and a response surface analysis was conducted with 3 factors and 3 levels, a total of 17 experimental points, based on the yield of L-arginine as the response value, as shown in Table 2.

\section{Results and Analysis}

\subsection{Results of signal factor experiment}

The effect of four single factors on the yield of L-arginine in fermentation broth is shown in Fig.1. In each single-factor experiment, when the glucose was $75 \mathrm{~g} / \mathrm{L}$, urea was $7 \mathrm{~g} / \mathrm{L}$ and soy peptone was 6 $\mathrm{g} / \mathrm{L}$, the concentration of L-arginine in fermentation broth reached the maximum respectively. While analysis results of single-factor experiment on calcium carbonate showed that the differences between different concentrations of calcium carbonate and yields of L-arginine in fermentation broth were not significant (at the level of 0.05).
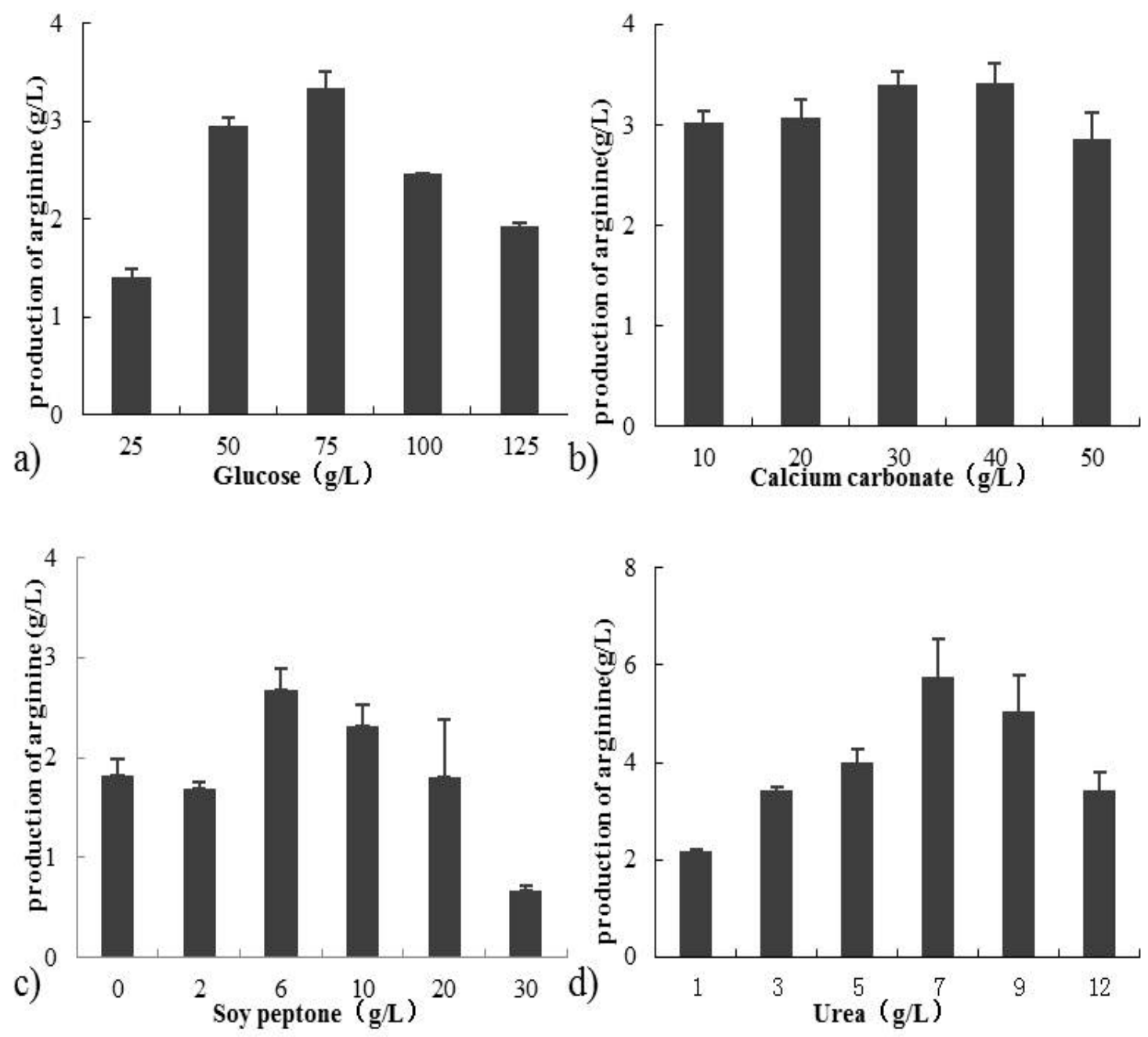

Fig.1 Effect of different factors of different concentrations on the yield of $L$-arginine

2.2 Response surface analysis

2.2.1 Experimental design and results

Through results of single-factor experiment, the concentration in the selected central point was $80.0 \mathrm{~g} / \mathrm{L}$ of glucose, $7.0 \mathrm{~g} / \mathrm{L}$ of soy peptone and $8.0 \mathrm{~g} / \mathrm{L}$ of urea respectively. According to 
Box-Bohnken' s experimental design, based on the yield of L-arginine as the response value, the experimental design and results of response surface are shown in Table 2.

\begin{tabular}{llllc}
\multicolumn{4}{c}{ Table 2 Experimental design and responses of Box-Behnken design } \\
\hline STD & A: & B: Urea(g/L) & C: Soy peptone(g/L) & R: Yield of $L$-arginine $(\mathrm{g} / \mathrm{L})$ \\
& Glucose(g/L) & & & \\
\hline 1 & 80.00 & 8.00 & 7.00 & 10.27 \\
2 & 110.00 & 8.00 & 10.00 & 8.21 \\
3 & 50.00 & 8.00 & 10.00 & 4.37 \\
4 & 80.00 & 8.00 & 7.00 & 9.42 \\
5 & 50.00 & 8.00 & 4.00 & 5.21 \\
6 & 110.00 & 6.00 & 7.00 & 6.20 \\
7 & 80.00 & 6.00 & 10.00 & 8.69 \\
8 & 80.00 & 6.00 & 4.00 & 8.94 \\
9 & 110.00 & 8.00 & 4.00 & 3.06 \\
10 & 80.00 & 8.00 & 7.00 & 8.49 \\
11 & 80.00 & 8.00 & 7.00 & 11.97 \\
12 & 80.00 & 10.00 & 4.00 & 3.46 \\
13 & 80.00 & 10.00 & 10.00 & 9.59 \\
14 & 110.00 & 10.00 & 7.00 & 2.56 \\
15 & 80.00 & 8.00 & 7.00 & 3.15 \\
16 & 50.00 & 10.00 & 7.00 & 6.44 \\
17 & 50.00 & 6.00 & 7.00 & \\
\hline
\end{tabular}

2.2.2 Quadratic regression fit and variance analysis

Based on the yield of L-arginine as the response value, according to results in Table 2, a multiple regression analysis was conducted on results in Table 2 using Design Expert 8.0.6 software. A regression equation was obtained:

Yield of L-arginine=10.05-1.39B+1.27C+1.50AC+1.59BC-3.90A2-145B2-0.93C2

A variance analysis of this model is shown in Table 3. From Table 3, P value in the model was 0.0018 , indicating that this quadratic regression model was significant. $\mathrm{P}$ value of lack of fit was 0.7776, indicating lack of fit in this model was not significant. A reliability analysis of this model is shown in Table 4 . The correlation coefficient was $\mathrm{R} 2=0.9377$, suggesting this quadratic regression model can better reflect the change of response values. This model and actual test fit well. There was a high correlation between predicted and actual values. It can be used for the theoretical prediction of L-arginine fermentation.

Table 3 Variance analysis of the regression equation

\begin{tabular}{ccccccc}
\hline \multicolumn{7}{c}{ Table 3 Variance analysis of the regression equation } \\
$\begin{array}{c}\text { Source of } \\
\text { Variation }\end{array}$ & Quadratic Sum & Degree of Freedom & Mean Square & $\begin{array}{c}\mathrm{F} \\
\text { Value }\end{array}$ & $\begin{array}{c}\mathrm{P} \\
\text { Value }\end{array}$ & \\
\hline Model & 129.80 & 9 & 14.42 & 11.96 & 0.0018 & significant \\
A-glucose & 0.024 & 1 & 0.024 & 0.020 & 0.8910 & $*$ \\
B-urea & 15.35 & 1 & 15.35 & 12.73 & 0.0091 & $*$ \\
C-soy peptone & 12.97 & 1 & 12.97 & 10.75 & 0.0135 & $*$ \\
AB & 0.15 & 1 & 0.15 & 0.13 & 0.7338 & $*$ \\
AC & 8.95 & 1 & 8.95 & 7.43 & 0.0295 & $*$ \\
BC & 10.15 & 1 & 10.15 & 8.42 & 0.0229 & $*$ \\
A $^{2}$ & 64.21 & 1 & 64.21 & 53.25 & 0.0002 & $*$ \\
B $^{2}$ & 8.88 & 1 & 8.88 & 7.36 & 0.0300 & $*$ \\
C $^{2}$ & 3.65 & 1 & 3.65 & 3.03 & 0.1252 & \\
Residual & 8.44 & 7 & 1.21 & & & \\
Lack of fit & 1.85 & 3 & 0.62 & 0.37 & 0.7776 & not significant \\
Pure error & 6.59 & 4 & 1.65 & & & \\
Total variation & 138.24 & 16 & & & & \\
\hline
\end{tabular}

\begin{tabular}{llll}
\multicolumn{3}{c}{ Table 4 Fit statistics for $\mathbf{Y}$} \\
\hline SD & 0.98 & $\mathrm{R}^{2}$ & 0.9377 \\
Mean & 7.09 & Adj R $^{2}$ & 0.8892 \\
Variable Coefficient & 13.79 & Pred R & 0.8219 \\
\hline
\end{tabular}

2.2.3 Seeking the optimal concentration of medium

3 factors in the above model equation and effect of their interaction on the response value can be seen visually through 3D plots of response surface in Figs 2, 3 and 4. From these plots, through further analysis with software, the maximum yield of L-arginine was $10.58 \mathrm{~g} / \mathrm{L}$. The corresponding 
optimal concentration was $84.44 \mathrm{~g} / \mathrm{L}$ of glucose, $7.83 \mathrm{~g} / \mathrm{L}$ of urea and $9.18 \mathrm{~g} / \mathrm{L}$ of soy peptone respectively.

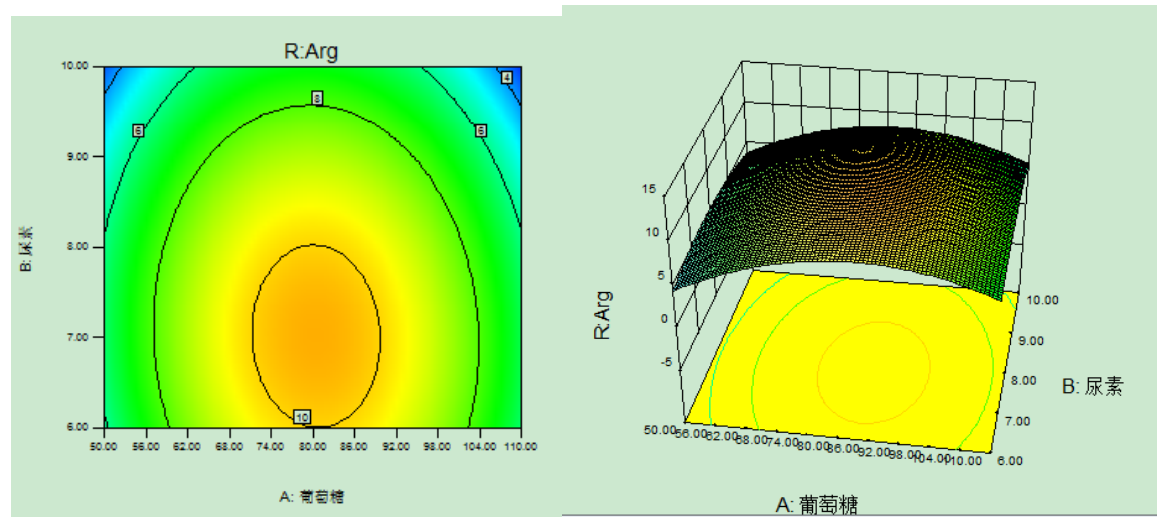

Fig.2 Response surface plot and contour maps of glucose and urea
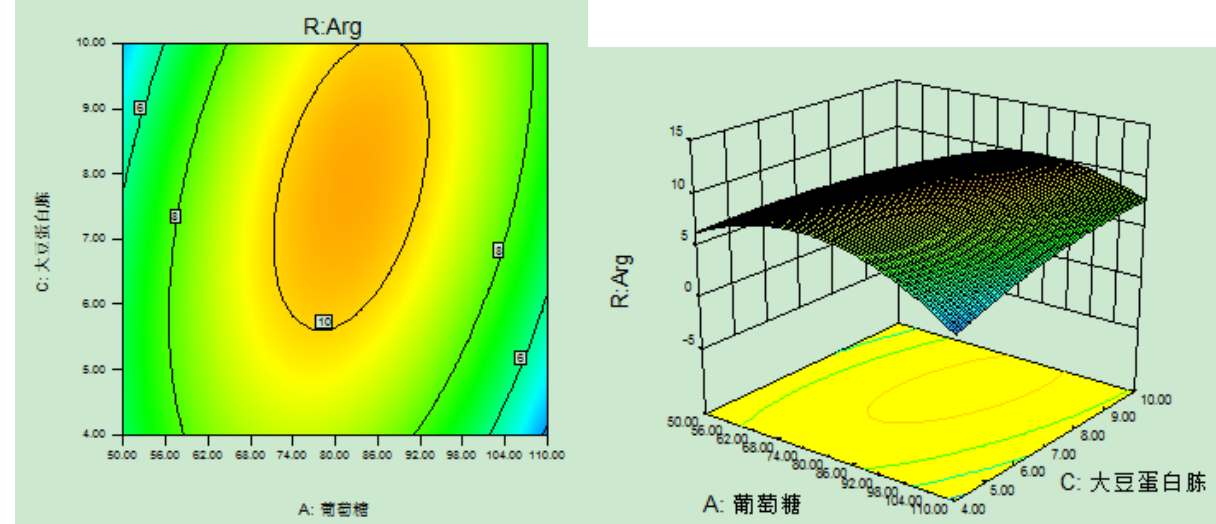

Fig.3 Response surface plot and contour maps of glucose and soya peptone

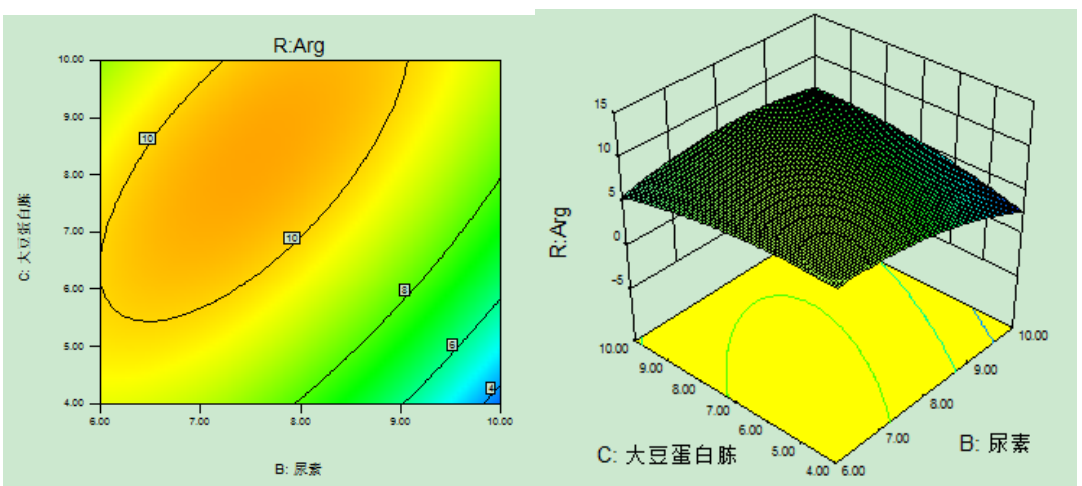

Fig.4 Response surface plot and contour maps of urea and soya peptone

\subsection{Model validation}

In order to validate the accuracy and effectiveness of this model, for the sake of expedience in experiments, eventually the concentrations were identified as follows: $80.0 \mathrm{~g} / \mathrm{L}$ of glucose, $8.0 \mathrm{~g} / \mathrm{L}$ of urea and $9.0 \mathrm{~g} / \mathrm{L}$ of soy peptone. The predicted optimum medium and initial medium were tested by shaking flask. Results showed that the average yield of L-arginine in fermentation broth under the initial condition was $3.65 \mathrm{~g} / \mathrm{L} /$. While the average yield of L-arginine in fermentation broth under the optimum condition was $10.77 \mathrm{~g} / \mathrm{L} /$. The yield of L-arginine was increased by $195.07 \%$, which was relatively close to the predicted value, showing that the regression model can truly reflect the effect of all factors on L-arginine production by fermentation.

In addition, the recombinant bacteria 14067-T18-argB-E-lysE, 14067-T18-argJBD-lysE and 14067-T18-argB-14-21 of several C.glutamicum constructed in this lab were fermented by shaking flask. The measured yields of L-arginine in their fermentation broth were significantly higher than before optimization, $193.49 \%$, $46.92 \%$ and $154.53 \%$ respectively. 


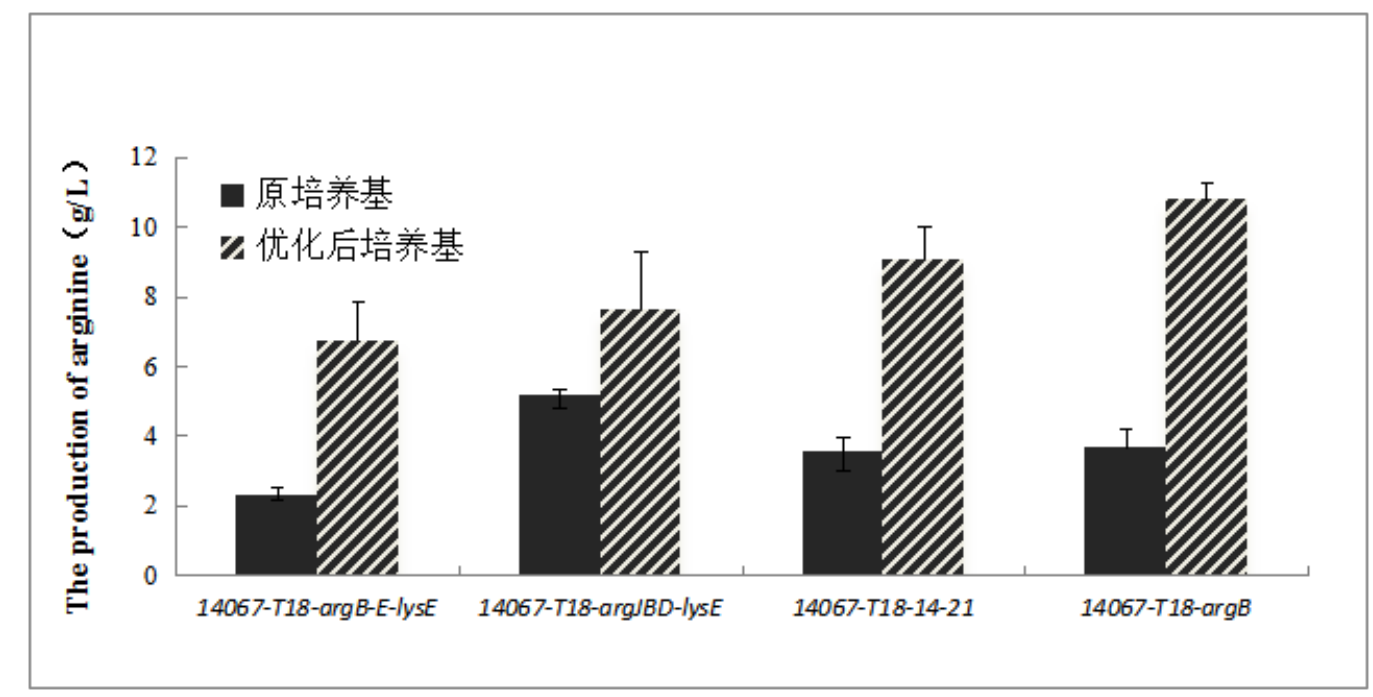

Fig.5 The production of $L$-arginine in optimum condition and initial condition fermentation of different recombinant bacteria

\section{Conclusion}

Carbon source provides energy required for the growth and reproduction of microbes and carbon element needed by synthetic bacteria. Also, glucose is the most common carbon source and an important affecting factor for the yield of L-arginine. If initial glucose is too low, substrate may be insufficient and reduce the yield of metabolites. If initial glucose is too high, substrate inhibition may occur, which is adverse to the growth of bacteria and fermentation. As a $\mathrm{pH}$ buffer, calcium carbonate can balance $\mathrm{pH}$ value in fermentation broth. In the process of L-arginine fermentation, with the genesis and on-going accumulation of L-arginine in fermentation broth, $\mathrm{pH}$ value in fermentation broth will constantly decrease. Calcium carbonate can adjust $\mathrm{pH}$ while supplying Ca2+. Nitrogen source is the source of synthetic mycoprotein, nucleic acid and other nitrogenous substance, as well as amino and guanido of L-arginine. Since nitrogen content in the fermented L-arginine molecules was as high as $32.15 \%$, there must be a higher proportion of nitrogen source in fermentation medium to meet the needs of growth of bacteria and L-arginine synthesis. Therefore, an appropriate amount of urea is very crucial for the improvement of the yield of L-arginine. And soy peptone, as a sustained-release nitrogen source of fermentation medium, is rich in growth factors and various amino acids. It can provide sufficient nutrition for the growth of bacteria, making bacteria thrive and benefiting the large accumulation of L-arginine.

This experiment adopts single-factor optimization and finally determines three factors that have a significant impact on the yield of L-arginine: glucose, urea and soy peptone. On this basis, Box-Bohnken's experimental designed is employed, to obtain the optimal model by solving the regression equation. The levels of various factors are $80 \mathrm{~g} / \mathrm{L}$ of glucose, $8.0 \mathrm{~g} / \mathrm{L}$ of urea and $9.0 \mathrm{~g} / \mathrm{L}$ of soy peptone. By validating the model, the predicted value of model is close to the average of validation test. Under the optimum condition, the yield of L-arginine increased by $195.07 \%$. With respect to the reasons of increase, there are two hypotheses. One is that the glucose concentration in the initial medium is high and inhibits the growth of bacteria. The other is that the optimum medium reduces the carbon nitrogen ratio in the initial medium, making the yield of L-arginine increase significantly.

Since all results of this experiment are obtained by shaking flask, it is difficult to achieve dissolved oxygen, $\mathrm{pH}$, glucose addition and other conditions in the process of fermentation. In order to further control the fermentation process and increase the yield of L-arginine, it is imperative to control fermentation process in a fermentor. Meanwhile, we can also seek reasons for the increase of yield of L-arginine in subsequent experiments. 


\section{References}

[1]Wu G, Bazer FW, Davis TA, et al. Arginine metabolism and nutrition in growth, health and disease[J]. Amino Acids, 2009,37(1): 153-168

[2]Barbul A. Arginine: biochemistry, physiology, and therapeutic implications[J]. Journal of Parenteral and Enteral Nutrition, 1986, 10(2): 227-238.

[3]Yan Hongbo, Wang Wei, Li Lingdi,et.al. Research Progress of the Arginine Biosynthetic Pathway in Prokaryotic Cells[J] BIOTECHNOLOGY BULLETIN,201531(1):21-28

[4]ZHOU Dong , TANG Zhiru, FENG Zemeng,et.al. Breeding of L-arginine-producing Strain and Optimization of Fermentation Conditions [J]. Research of Agricultural moderization,2010,06:733-737

[5]Ginesy M, Belotserkovsky J, Enman J, et al. Metabolic engineering of Escherichia coli for enhanced arginine biosynthesis [J]. Microbial cell factories, 2015, 14(1): 29.

[6]Park S H, Kim H U, Kim T Y, et al. Metabolic engineering of Corynebacterium glutamicum for L-arginine production[J]. Nature communications, 2014, 5.

[7]ZHENG Hao, CHENG Xian-Long, WEI Feng, et al. Pre-column derivatization RP-HPLC determation of 16 amino acids in omphalia lapidescens Schroet [J]. Chin. J.Pharm. Anal., 2011, 31(9): 1631-1635 